\title{
Application of next-generation sequencing to identify mitochondrial mutations: Study on m.7511T $>C$ in patients with hearing loss
}

\author{
URSZULA LECHOWICZ ${ }^{1}$, AGNIESZKA POLLAK ${ }^{1}$, AGNIESZKA FRĄCZAK ${ }^{1}$, MAŁGORZATA RYDZANICZ ${ }^{2}$, \\ PIOTR STAWIŃSKI ${ }^{1}$, ARTUR LORENS $^{3}$, PIOTR H. SKARŻYŃSKI ${ }^{4-6}$, HENRYK SKARŻYŃSKI $^{7}$, \\ RAFAŁ PŁOSKI ${ }^{2 *}$ and MONIKA OŁDAK ${ }^{1 *}$
}

\author{
${ }^{1}$ Department of Genetics, World Hearing Center, Institute of Physiology and Pathology of Hearing, 02-042 Warsaw; \\ ${ }^{2}$ Department of Medical Genetics, Center for Biostructure, Medical University of Warsaw, 02-106 Warsaw; \\ ${ }^{3}$ Department of Implants and Auditory Perception; ${ }^{4}$ World Hearing Center, Institute of Physiology and Pathology of Hearing, \\ 02-042 Warsaw; ${ }^{5}$ Department of Heart Failure and Cardiac Rehabilitation, Second Faculty, Medical University of Warsaw, \\ 03-242 Warsaw; ${ }^{6}$ Institute of Sensory Organs, $05-830$ Nadarzyn; ${ }^{7}$ Oto-Rhino-Laryngology Surgery Clinic, \\ Institute of Physiology and Pathology of Hearing, 02-042 Warsaw, Poland
}

Received June 29, 2017; Accepted October 24, 2017

DOI: $10.3892 / \mathrm{mmr} .2017 .8064$

\begin{abstract}
Interruptions in the activity of mitochondria induced by mutations in the mitochondrial genome (mtDNA) can be the source of numerous diseases including hearing loss (HL). One of the mitochondrial variants responsible for HL is the $\mathrm{m} .7511 \mathrm{~T}>\mathrm{C}$ mutation located in the mitochondrially encoded tRNA serine 1 (UCN) gene. Next-generation sequencing was used to search for the HL mutations in the whole mtDNA of 2 patients with maternal inheritance and real time-polymerase chain reaction was applied for population screening of the m.7511T $>C$ mutation in a group of 1,644 patients with HL Sequencing of the whole mtDNA in 2 probands revealed a homoplasmic m.7511T $>C$ mutation. Inheritance of the m.7511T $>\mathrm{C}$ mutation has been confirmed in examined matrilineal relatives in both families. The mean age of HL onset was 14.1 years old with the mean degree of HL equaling $74.8 \mathrm{~dB}$. A large-scale search for the m.7511T $>C$ mutation among the patients with HL established the frequency of the m.7511T $>\mathrm{C}$
\end{abstract}

Correspondence to: Dr Monika Ołdak, Department of Genetics, World Hearing Center, Institute of Physiology and Pathology of Hearing, 10 M. Mochnackiego, 02-042 Warsaw, Poland

E-mail:m.oldak@ifps.org.pl

Professor Rafał Płoski, Department of Medical Genetics, Center for Biostructure, Medical University of Warsaw, 3c Pawinskiego, 02-106 Warsaw, Poland

E-mail: rploski@wp.pl

*Contributed equally

Key words: hearing loss, m.7511T $>\mathrm{C}$ mutation, mitochondrially encoded tRNA serine 1 (UCN), mitochondrial, maternal inheritance mutation at $0.12 \%$ among Polish patients with HL. In conclusion, this first report on central European patients harboring the $\mathrm{m} .7511 \mathrm{~T}>\mathrm{C}$ mutation reveals that the m.7511T $>\mathrm{C}$ may be important when diagnosing patients with maternally inherited HL.

\section{Introduction}

Hundreds to thousands of mitochondria are present on average in a human cell. Each of the mitochondrion contains several copies of inherited maternally 16 569-base pair, circular, double-stranded DNA molecule (mtDNA). The main role of mitochondria is to provide energy to the cell in a process of oxidative phosphorylation, running through specialized complexes located in the mitochondrial electron transport chain. Approximately $10 \%$ of proteins necessary for mitochondrial activity (13 respiratory chain peptides, 22 transfer RNAs and 2 mitochondrial ribosomal RNAs) are encoded by its own genome. The majority of proteins found in mitochondria are encoded by the nuclear genes $(1,2)$. Disturbances in effective functioning of mitochondria resulting from mutations in the mitochondrial genome can lead to various disorders including hearing loss (HL). It can occur as an isolated feature or a part of a genetic syndrome, e.g., mitochondrial encephalomyopathy, lactic acidosis, and stroke-like episodes (MELAS), myoclonic epilepsy with ragged-red fibers (MERFF) or Kearns-Sayre syndrome (KSS), which is a mitochondrial myopathy, characterized by ptosis and ophthalmoplegia and other symptoms such as proximal muscle weakness, cerebellar ataxia, diabetes mellitus and/or endocrinopathies (3).

Identifying the genetic background of isolated, sensorineural hearing loss (SNHL) is challenging because deafness may result from mutations in many different genes (4). In patients with isolated, prelingual and recessive SNHL mutations in the deafness, autosomal recessive 1 (DFNB1) 
locus, containing the gap junction protein beta 2 (GJB2) and the gap junction protein beta $6(G J B 6)$, are responsible for about $50 \%$ of cases $(4,5)$. Conversely, isolated, congenital maternally inherited SNHL is as rare as $1 \%$ (6) but the prevalence of postlingual, maternally inherited HL has been estimated even up to $30 \%$ (7-9). The most common mtDNA mutations in the Polish HL patients are m.1555A $>\mathrm{G}$ in the $M T-R N R 1$ gene and $\mathrm{m} .3243 \mathrm{~A}>\mathrm{G}$ in the MT-TL1 gene with the prevalence of approximately $1 \%$ for each of them $(10,11)$. The vast majority of mtDNA mutations cause postlingual, bilateral and symmetrical HL, which may progress over time. Along with HL, tinnitus and/or vertigo are also observed in many individuals (12-15). Mutations in mtDNA related to HL are homoplasmic (pure population of mutated mtDNA) but various levels of heteroplasmy (mixture of wild-type and mutant mtDNA) are also found.

MT-TS1 gene (MIM 590080) encoding tRNASer ${ }^{(\mathrm{UCN})}$, a small 69 nucleotide RNA represents another HL-related hot spot in the mitochondrial genome. Two mutations of this gene, i.e., m.7510T $>\mathrm{C}$ and $\mathrm{m} .7511 \mathrm{~T}>\mathrm{C}$ are located in the acceptor arm of the tRNA molecule. They disrupt the highly conserved structure of the acceptor stem of the tRNASer ${ }^{(\mathrm{UCN})}$. The m.7511T $>C$ mutation reduces by approximately $80 \%$ the level of tRNA synthesis, strongly affecting the mitochondrial protein translation (16) leading to an impaired oxidative phosphorylation. Heretofore, $\mathrm{m} .7511 \mathrm{~T}>\mathrm{C}$ mutation has been identified only in $7 \mathrm{HL}$ families worldwide $(1,17-24)$.

Since introduction of next-generation sequencing (NGS), analysis of the whole mitochondrial DNA is no longer labor intensive. Having a complete sequence of the mitochondrial genome, the involvement of other mtDNA mutations in the development of HL can be excluded and patients can obtain an accurate result of mitochondrial genetic testing.

In this study we have identified the $\mathrm{m} .7511 \mathrm{~T}>\mathrm{C}$ mutation in two unrelated HL families using NGS of the whole mtDNA. This is the first report on Polish patients harboring m.7511T $>C$, which includes their detailed audiological characteristics as well as the frequency of this mutation among more than 1,600 Polish HL individuals.

\section{Materials and methods}

Patients. Two DNA samples derived from unrelated probands (family A and B; both of Caucasian origin) with a maternal type of HL inheritance were selected for NGS sequencing of the whole mtDNA (Fig. 1). Mutations at the DFNB1 locus for which HL patients at the Department of Genetics (Institute of Physiology and Pathology of Hearing, Warsaw, Poland) are routinely tested according to the EMQN recommendations (25) and mitochondrial DNA mutations (m.1555A $>$ G, m.3243A>G) as well as major pre- and perinatal HL risk factors (i.e., severe prematurity, congenital rubella, mumps or cytomegalovirus infection, severe neonatal hyperbilirubinemia) were not identified among the surveyed members from both families.

For screening of the m.7511T $>\mathrm{C}$ mutation a group of 1,644 unrelated HL patients of Caucasian origin, with a various degree of HL and without common GJB2 mutations (c.35delG, c.334_335delAA, c.358_360delGAG, c.167delT, c.313_326del) was selected. Based on the age of onset (AO), the group was divided into subgroups of 600 patients with prelingual (<6 years) and 1,044 patients with postlingual ( $>6$ years) $\mathrm{HL}$.

All procedures performed in studies involving human participants were in accordance with the ethical standards of the institutional and/or national research committee and with the 1964 Helsinki declaration and its later amendments or comparable ethical standards. The study was approved by the local Ethics Committee (IFPS:/KB/04/2012). Informed consent was obtained from all individual participants included in the study.

Audiological assessment in patients with the m.7511T $>C$ mutation. Hearing levels were determined by pure-tone audiometry at frequencies of $0.5,1,2,4$ and $8 \mathrm{kHz}$ and classified according to the pure tone average (PTA) as mild (21-40 dB HL), moderate (41-70 dB HL), severe (71-90 dB HL) and profound (>90 dB HL) (26). Temporal bone computer tomography (CT) or magnetic resonance imaging (MRI) scans were obtained preoperatively from patients with cochlear implants (CI). Preoperatively, and at intervals of 1 , 2 and 3 years postimplantation, patients were evaluated using pure-tone audiometry on both ears in the unaided condition and were tested for Polish monosyllabic word recognition (prerecorded on compact disc) and presented at $70 \mathrm{~dB}$ Sound Pressure Level (SPL) in quiet and in $10 \mathrm{~dB}$ signal-to-noise ratio (SNR) speech spectrum noise (27). Forty words (2 lists of 20 words) were presented from a loudspeaker placed $1.5 \mathrm{~m}$ from the subject in an Industrial Acoustics Company Inc. (Winchester, UK) double-walled booth. Lists were chosen at random, with no list being used twice with the same subject. Preoperatively, subjects were tested with two hearing aids (HAs). Postoperatively, subjects were tested using the CI with the contralateral ear HA.

DNA isolation. DNA was isolated from peripheral blood by a standard salting out method (28).

Next-generation and Sanger sequencing of $m t D N A$. In the first step two overlapping amplicons of the whole mtDNA ( $\sim 9 \mathrm{~kb}$ each) were produced by a long range PCR using Takara LA Taq Hot Start polymerase (Takara Bio Inc., Kusatsu, Japan) with the specific primers: Amt123F, 5'CTT TGATTCCTGCCTCATCC; Amt8663R, 5'GGGTGGTGA TTAGTCGGTTG; Bmt8467F, 5'CCTACCTCCCTCACC AAAGC; and Bmt824R, 5'ATCACTGCTGTTTCCCGTGG designed with Primer3 software (http://bioinfo. ut.ee/primer3-0.4.0/primer3/) (29,30) based on the mtDNA revised Cambridge Sequence (rCRS; GenBank accession no. NC_012920). PCR was performed under the following conditions: An initial 2 min incubation at $94^{\circ} \mathrm{C}$ was followed by 40 cycles of PCR with $10 \mathrm{sec}$ of denaturation at $98^{\circ} \mathrm{C}$ and $18 \mathrm{~min}$ of annealing and extension at $68^{\circ} \mathrm{C}$. The reaction was completed by 1 cycle of final extension at $72^{\circ} \mathrm{C}$ for $10 \mathrm{~min} ; 1.5 \mu \mathrm{l}$ of PCR product was analyzed on $1.5 \%$ agarose gel with $1 \mathrm{~kb}$ plus DNA ladder (Invitrogen; Thermo Fisher Scientific, Inc., Waltham, MA, USA). Next, DNA libraries were prepared with the NexteraXT Sample preparation kit according to the manufacturer's instructions (Illumina Inc., San Diego, CA, USA) and a paired-end index sequencing ( 2 x150 bp) was performed on the MiSeq sequencer (Illumina Inc.). The obtained data were 


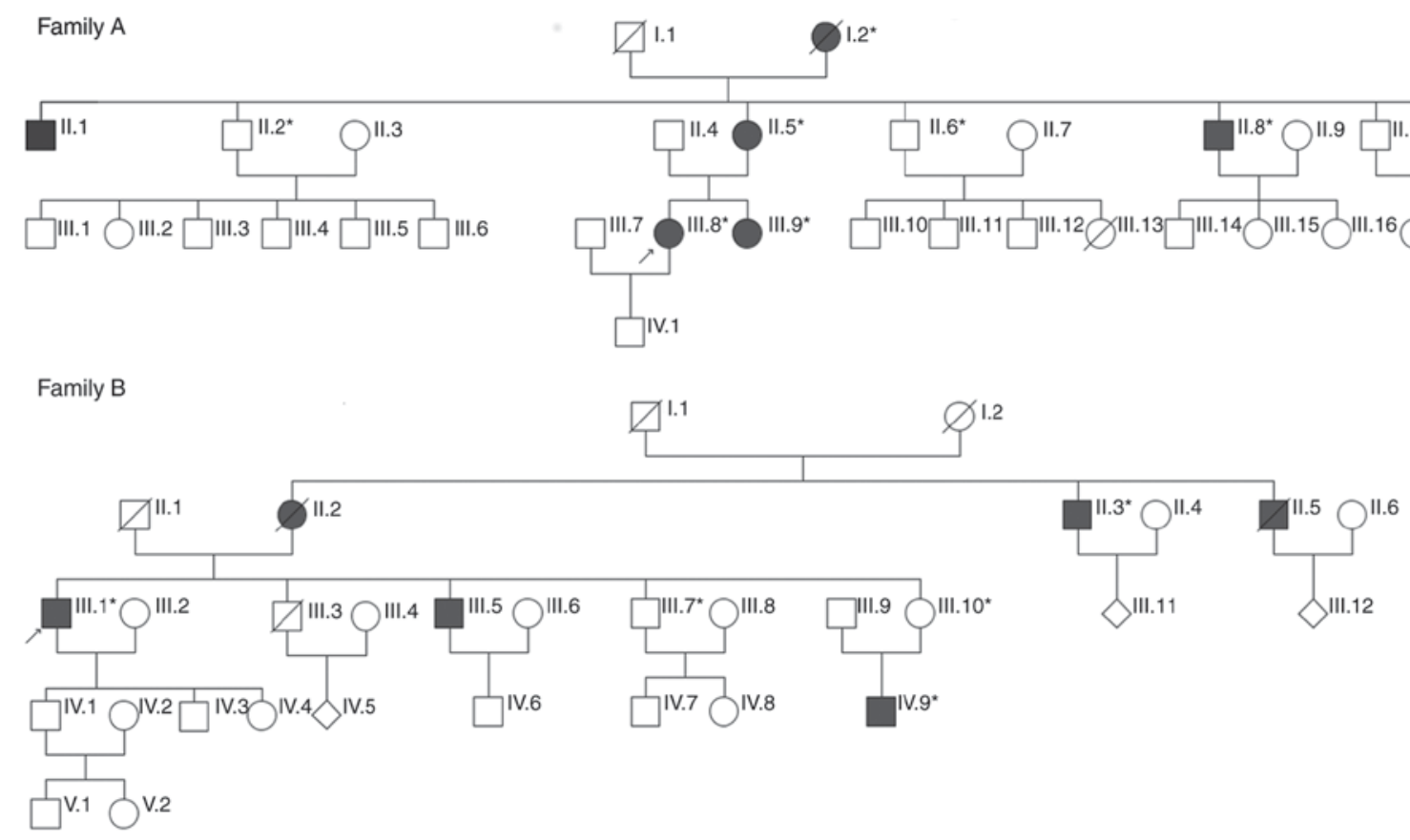

Figure 1. Pedigrees of Family A and B. Circles represent females, squares represent males and diamonds depict individuals of undetermined gender. The filled symbols indicate affected individuals and asterisks indicate subjects tested for m.7511T $>\mathrm{C}$ mutation. Probands are marked with arrows and symbols with a diagonal line through it indicate deceased family members.

analyzed by the CASAVA software (Illumina Inc.) for demultiplexing and FASTQ file generation. Reads were aligned to rCRS using the Burrows-Wheeler Aligment Tool and processed further by the Picard and Genome Analysis Toolkit (31-33). Population frequency of the identified variants and the corresponding phenotypic information were derived from the online mitochondrial genome database (http://www.mitomap.org) and converted to the MS Excel format for final manual analyses (34). NGS results were inspected with Integrative Genomics Viewer (IGV) (35). The average coverage for both samples was above 400x. Variants described in the MitoMap database (http://www.mitomap.org/MITOMAP) as pathogenic were selected for primary analysis. Next, variants with low population frequency in MitoMap and Human Mitochondrial Genome Database (http://www.mtdb.igp.uu.se/), non-synonymous or located in highly conserved and/or functionally important domains of tRNA or rRNA were considered as potentially pathogenic (36).

Presence of the identified mutations was confirmed by Sanger sequencing. PCR primers Mt7271F, 5'-GGCTCA TTCATTTCTCTAACAGC-3' and Mt7649R, 5'-GGGCGT GATCATGAAAGGTG-3' for the region encompassing m.7511T $>C$ were designed with the Primer 3 software (http://bioinfo.ut.ee/primer3-0.4.0/primer3/) based on rCRS. PCR was performed under the following conditions: an initial 5-min incubation at $95^{\circ} \mathrm{C}$ was followed by 32 cycles of PCR with $30 \mathrm{sec}$ of denaturation at $95^{\circ} \mathrm{C}, 55 \mathrm{sec}$ of annealing at $56^{\circ} \mathrm{C}$ and $1 \mathrm{~min}$ of extension at $72^{\circ} \mathrm{C}$. The reaction was completed by 1 cycle of final extension at $72^{\circ} \mathrm{C}$ for $10 \mathrm{~min}$.

Heteroplasmy level, penetration score and determination of haplogroups. Heteroplasmy level of m.7511T $>C$ was estimated based on the electropherograms from Sanger sequencing or IGV data. Penetration score was calculated as a number of
HL individuals among all matrilineal relatives in a family. Haplogroups were determined with HaploGrep 2 (v2.1.0) and Mitomaster tools $(37,38)$.

Real time PCR. Real time PCR screening for the m.7511T $>C$ mutation was performed on the Viia7 system (Life Technologies, Carlsbad, CA, USA) using an assay on demand containing a respective primer pair encompassing m.7511 and fluorescently-labelled TaqMan probes for the discrimination of m.7511T and C (Life Technologies). As a positive control DNA sample with a confirmed m.7511T $>$ C mutation was used.

Statistical analysis. Mutation frequency among different populations was compared by Chi-square statistics. Correlation between the heteroplasmy level and AO was determined with the Spearman Rank Correlation (Spearman's rho) test (39). The data was considered statistically significant at $\mathrm{P}<0.05$.

\section{Results}

Sequencing of the whole mtDNA in two probands from unrelated HL families with pedigrees suggesting maternal inheritance, revealed a homoplasmic m.7511T $>C$ mutation in the mitochondrial MT-TS1 gene (Fig. 2). Presence of the mutation was confirmed by Sanger sequencing (data not shown).

To investigate the segregation of m.7511T $>\mathrm{C}$ with $\mathrm{HL}$, available DNA samples from the family members have been Sanger sequenced. Inheritance of $\mathrm{m} .7511 \mathrm{~T}>\mathrm{C}$ has been confirmed over generations in matrilineal relatives in both families. From nine DNA samples collected from family A seven had the m.7511T $>C$ mutation in a homoplasmic state, in one $50 \%$ heteroplasmy was detected and the other one (A.II.10) who did not carry the mutation also did not have HL 
Table I. Detailed clinical characterization of patients harboring the m.7511T $>$ C mutation and heteroplasmy level among patients.

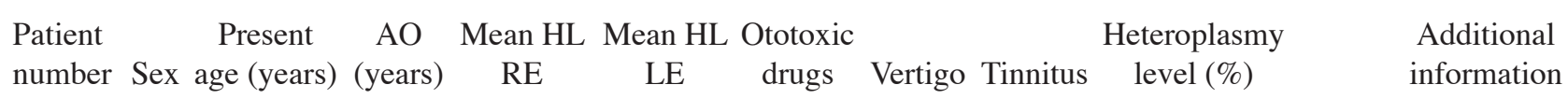

\begin{tabular}{|c|c|c|c|c|c|c|c|c|c|}
\hline A.I.2 & $\mathrm{F}$ & 87 & 3.5 & 86 & 113 & - & - & - & 50 \\
\hline A.II.2 & $\mathrm{M}$ & 57 & - & - & - & - & - & - & 100 \\
\hline A.II.5 & $\mathrm{F}$ & 55 & 37 & 84 & 85 & + & + & + & 100 \\
\hline
\end{tabular}

\begin{tabular}{|c|c|c|c|c|c|c|c|c|c|c|}
\hline & & & & & & & & & & \\
\hline A.II.6 & M & 54 & - & - & - & - & - & - & 100 & \\
\hline A.II.8 & M & 51 & 40 & 57 & 59 & - & - & - & 100 & \\
\hline A.II.12 & $\mathrm{F}$ & 47 & 2.5 & 30 & 48 & - & - & + & 100 & \\
\hline A.III.8 & $\mathrm{F}$ & 28 & 3 & 69 & 65 & - & - & - & 100 & HA \\
\hline A.III.9 & $\mathrm{F}$ & 34 & 0 & 85 & 79 & - & + & - & 100 & $\begin{array}{l}\mathrm{CI}(\mathrm{RE}) \text { and HA }(\mathrm{LE}) ; \\
\text { preoperative CT of temporal } \\
\text { bones-no abnormalities; }\end{array}$ \\
\hline B.II.3 & M & 76 & 27 & 92 & 97 & - & - & - & 100 & HA \\
\hline B.III.1 & M & 66 & 7 & 68 & 79 & - & + & + & 100 & \\
\hline B.III.7 & M & 64 & - & - & - & - & - & - & 100 & \\
\hline B.III.10 & $\mathrm{F}$ & 60 & - & - & - & - & - & + & 100 & \\
\hline B.IV.9 & M & 36 & 7 & nd & nd & - & - & - & 100 & \\
\hline
\end{tabular}
preoperative MRI of abnormalities

M, male; F, female; HL, hearing loss; nd, no data available; AO, age of onset; CI, cochlear implant; HA, hearing aids; RE, right ear; LE, left ear; CT, computer tomography; MRI, magnetic resonance imaging.
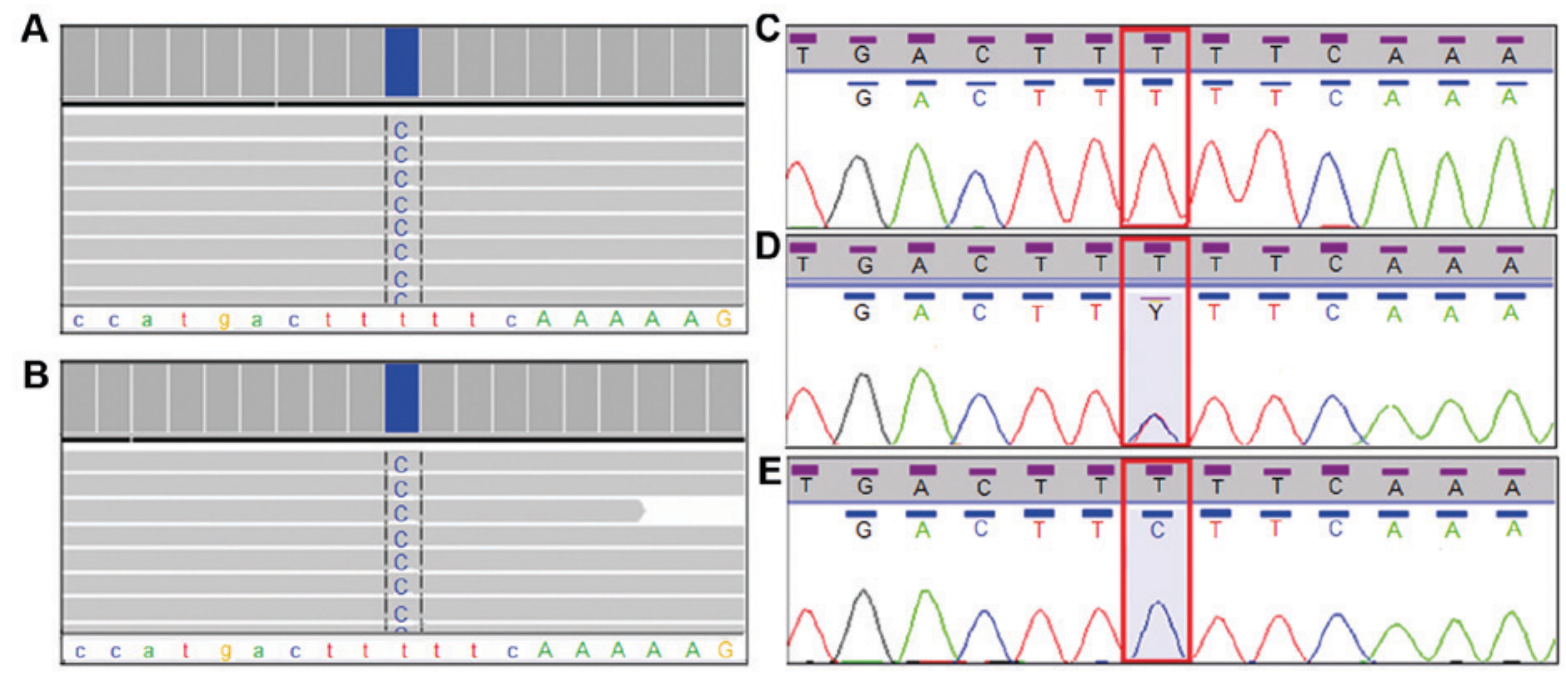

Figure 2. Identification of the m.7511T $>C$ mutation with $(A$ and $B$ ) next-generation sequencing and $(C-E)$ Sanger sequencing. Identification of $\mathrm{m} .7511 \mathrm{~T}>\mathrm{C}$ mutation (dark blue rectangle) in the proband from (A) family A and (B) family B. The IGV image of m.7511T $>$ C is shown following whole mtDNA sequencing. The different heteroplasmy levels of the m.7511T>C mutation have also been displayed, with the representative Sanger sequencing results of $(C)$ wild-type m.7511T, (D) 50\% level of m.7511T >C heteroplasmy and (E) m.7511C homoplasmy in the mitochondrially encoded tRNA serine 1 (UCN) gene. The m.7511 position is contoured by a frame.

(Figs. 1 and 2). Among patients with m.7511T $>\mathrm{C}$ mutation in family A, HL was observed in six out of eight individuals and there was an interfamilial variability in its $\mathrm{AO}$ (0-40 years) and severity (from moderate to profound) (Table I). All tested matrilineal relatives from family B had a homoplasmic m.7511T $>C$ mutation. In three individuals postlingual HL has been diagnosed, while the remaining two carriers of the mutation did not suffer from HL.
Among the tested individuals in both families only patients with $\mathrm{m} .7511 \mathrm{~T}>\mathrm{C}$ had HL. The penetration score was calculated as $64 \%$ (7/11) for family A and 60\% (6/10) for family B, which makes an average value of $62 \%$ (13/21). The mean age of HL onset in the individuals was 14.1 years with the mean degree of HL being $74.8 \pm 20.3 \mathrm{~dB}$, which corresponds to a moderate HL. There was a weak, positive correlation between the level of heteroplasmy and $\mathrm{AO}$, but it was not statistically significant 

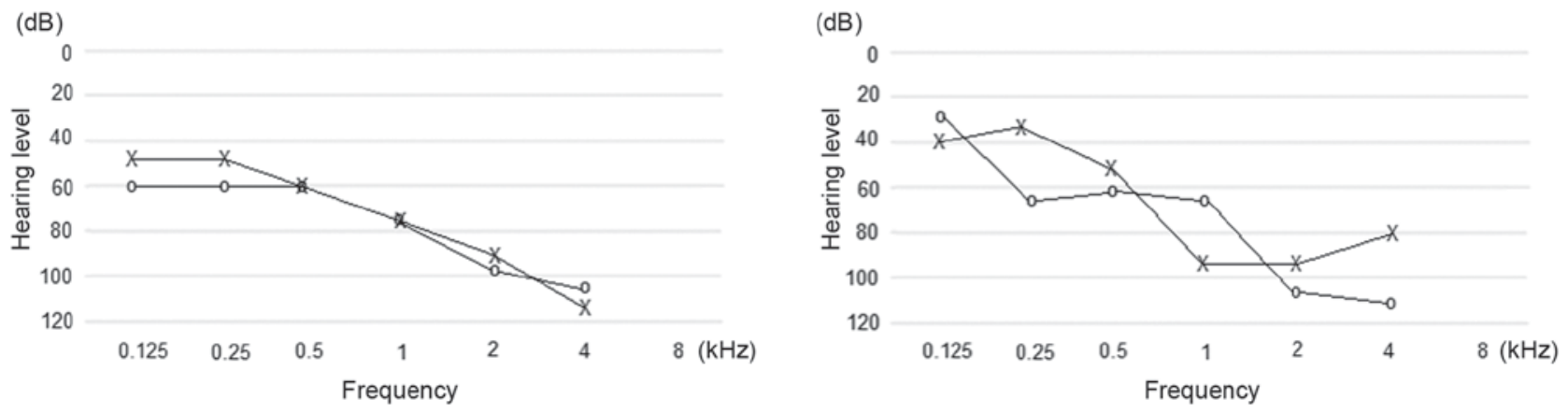

Figure 3. Audiograms of patients A.II.5 (left panel) and A.III.9 (right panel). Circles correspond to the right ear and X corresponds to the left ear.

$(\mathrm{R}=0.08, \mathrm{P}=0.84)$. Some patients in both families suffered from vertigo or/and tinnitus (Table I).

Some of the affected individuals are using HA or $\mathrm{CI}$ and HA as a treatment (Table I). In two patients with partial deafness (PD) $(40,41)$ (Fig. 3) cochlear implantation was successfully applied (42). No structural abnormalities were observed on the temporal bone CT scans. At the age of 51, patient A.II.5 received CI (Med-El Concerto Flex 24, Med-El, Austria) in the right ear (RE). Before surgery the monosyllabic speech discrimination in quiet and noise in the bilaterally best fitted HAs was 60 and $15 \%$ correct responses, respectively. According to the hearing preservation classification, acoustic hearing was preserved completely after implantation (43). Over a period of 3 years, monosyllabic word recognition increased from 60 to $95 \%$ under quiet conditions and from 15 to $40 \%$ under noisy conditions. Patient A.III.9 with bilateral mild-to-profound HL obtained CI [CI24RE (SRA); Cochlear, Melbourne, Australia] in the RE at the age of 18 years (44). Before surgery the monosyllabic speech discrimination in quiet and noise in the bilaterally best fitted HAs was 30 and $0 \%$, respectively. After implantation hearing was partially preserved. Over a period of 3 years, monosyllabic word recognition increased from 30 to $100 \%$ under quiet conditions and from 0 to $75 \%$ under noisy conditions. Both patients substantially benefited from CI.

Prevalence of m.7511T>C in the Polish HL population and in other populations. Searching for the m.7511T $>\mathrm{C}$ mutation among both subgroups of HL patients $(n=1644)$ did not identify any additional cases of m.7511T $>C$ mutation. Taking into account that our probands also fulfilled criteria for real time PCR screening, we have estimated the frequency of m.7511T $>C$ mutation at $0.12 \%(2 / 1646)$ among HL patients with excluded common GJB2 mutations.

We have compared our data to the Chinese HL and Japanese HL patients among whom the incidence of m.7511T $>\mathrm{C}$ was assessed at $0.04 \%(1 / 2651)(19)$ and $0.7 \%(1 / 140)(20)$, respectively and the difference was not statistically significant ( $\mathrm{P}=0.31$ for the Chinese and $\mathrm{P}=0.1$ for the Japanese cohorts, respectively).

Other mtDNA variants identified in whole mitochondrial sequencing. Results of the whole mtDNA sequencing in patients A.III.8 and B.III.1 have shown several other insignificant variants such as polymorphisms, synonymous or non-synonymous variants with a high prevalence in a general population. Having a list of all mtDNA variants for both patients we determined the haplogroup H44b for family A and the haplogroup $\mathrm{T} 2 \mathrm{~b}$ for family $\mathrm{B}$.

\section{Discussion}

Mitochondrial mutations are not routinely tested among HL patients and their incidence can be underestimated (13). A study aimed at detecting both, known as well as novel mtDNA variants related to HL, is important and may bring new insight into the pathogenesis of HL. The fact that common mtDNA mutations such as m.1555A $>\mathrm{G}$ and m.3243A $>\mathrm{G}$ were identified among Polish HL families with an unexpected high frequency $(\sim 1 \%)$ prompted us to further investigate other mtDNA mutations causing HL $(10,11)$. The presence of these two mutations was checked in our patients prior to their inclusion into the study. No reports describing the prevalence of m.7511T $>\mathrm{C}$ in the Polish population have been published previously and in studies from other populations the prevalence of the m.7511T $>C$ is low. Thus, we did not expect to detect this particular variant in our patients and proceeded with NGS.

The present study provides a clinical and genetic characterization of two Polish families with isolated SNHL due to m.7511T $>\mathrm{C}$ mutation. To date, the $\mathrm{m} .7511 \mathrm{~T}>\mathrm{C}$ mutation has been detected only in $7 \mathrm{HL}$ families (Table II) of African-American (24), French (18), Japanese $(17,20)$ and Chinese $(19,21)$ origin, but heretofore it has not been reported in HL patients from central Europe.

In this study we assessed for the first time the frequency of m.7511T $>\mathrm{C}$ in the group of Polish HL patients. Its frequency of $0.12 \%$ is not significantly different from that of $\mathrm{m} .7445 \mathrm{~A}>\mathrm{G}$ ( $0.4 \%, 1 / 250$ Polish nonsydromic HL patients) (45), which is considered a major HL causative mutation in the MT-TSI gene. Two other common mtDNA mutations in Polish HL patients are m.1555A $>\mathrm{G}$ in $M T-R N R 1$ detected at a frequency of $1.3 \%$ (20/1499) and m.3243A $>\mathrm{G}$ in MT-TL1 with a frequency of $1 \%$ $(16 / 1499)(10,11)$. Based on the results, the total percentage of mitochondrial HL-related mutations in Polish patients can be estimated at almost $3 \%$.

Various m.7511T $>\mathrm{C}$ penetration levels have been reported in respect to HL. It ranges from $30 \%$ in some French or Japanese families to $84 \%$ in the African-American pedigree $(17,18,20,24)$. In the Polish families the penetration rate was established at an average of $62 \%$. It is a rather high score, 


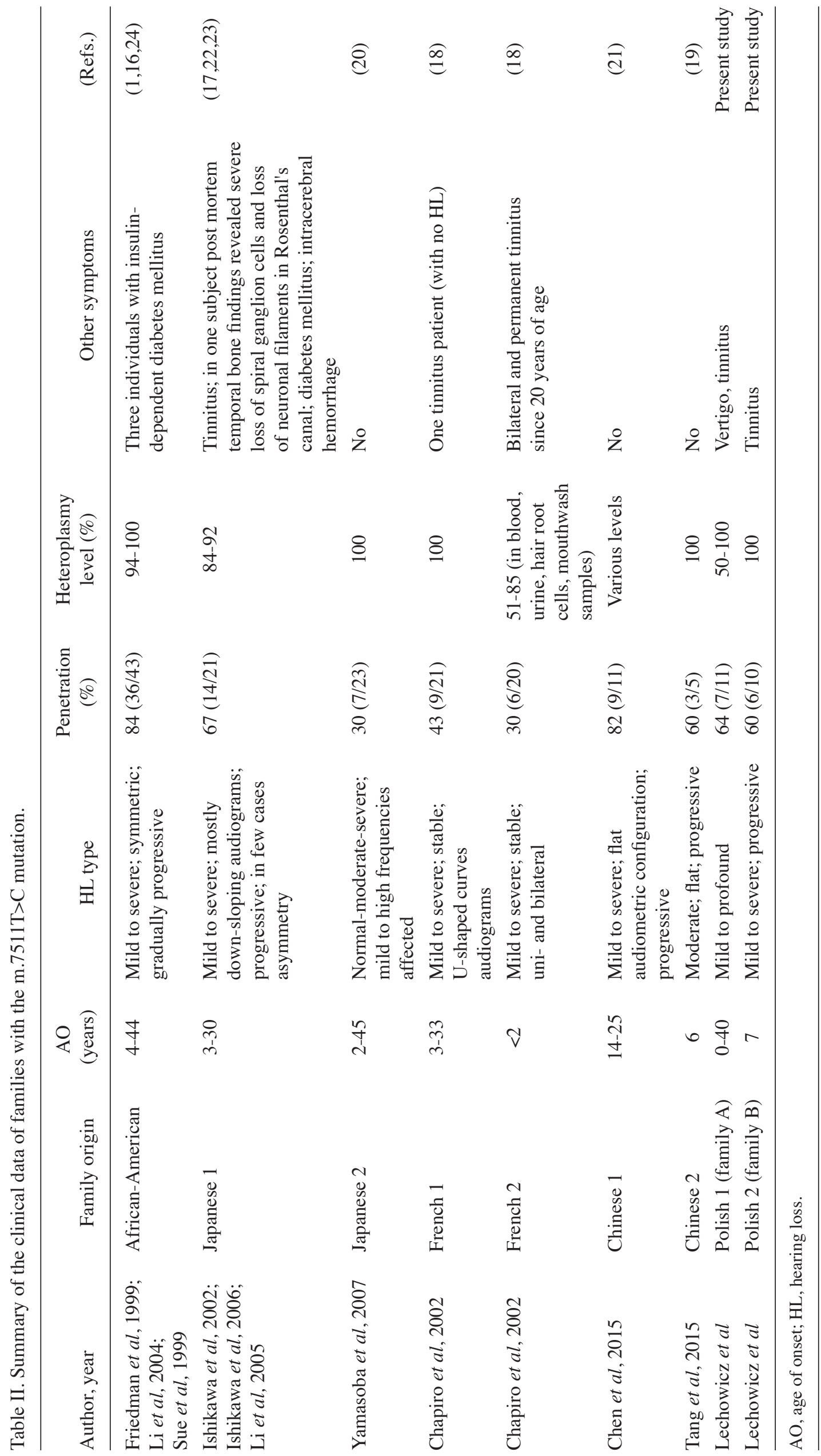


particularly when compared with other Caucasian families $(36.5 \%)$. It is not easy to explain such differences in penetration score, mainly because there is still a gap in our knowledge about the precise pathomechanisms of $\mathrm{m} .7511 \mathrm{~T}>\mathrm{C}$ mutation and/or coexistence of possible modifying factors.

MT-TS1 deafness mutations often occur in homoplasmy or high levels of heteroplasmy, suggesting a high heteroplasmic threshold for pathogenicity. Interestingly, in both Polish families we have observed patients with homoplasmic m.7511T $>C$ mutation, among whom no HL has been so far diagnosed (individuals: A.II.2, A.II.6, B.III.7, B.III.10; Table I). On the other hand, the subject A.I.2 who had an early onset and profound HL was found to have just $50 \%$ heteroplasmy level of the m.7511T $>C$ mutation. Sequencing of the entire mtDNA and determining that both our families belong to different mitochondrial haplogroups suggest that factors modifying the AO, degree and progression of HL are rather not located in mtDNA. Only in three previous reports of the m.7511T $>$ C mutation, the authors have sequenced the whole mtDNA, thereby excluding a direct role of other mtDNA variants or their co-involvement in the pathogenesis of HL. The results suggest that other polymorphisms and/or mutations in the nuclear genome as well as environmental factors may modulate the phenotypic variation $(18,24)$ but the triggering or modifying mechanisms still await elucidation.

It is known that some mutations in the mtDNA (e.g., m.1555A $>$ G) create an increased susceptibility to aminoglycosides, which results in ototoxicity and HL $(10,46)$. Except for one patient (Table I), none of the other studied individuals were treated with aminoglycosides. Thus, their role as a factor triggering $\mathrm{HL}$ in patients with $\mathrm{m} .7511 \mathrm{~T}>\mathrm{C}$ mutation can be excluded based on our and other studies $(1,18)$. There is a great variation in the $\mathrm{HL} \mathrm{AO}$ in our patients with $\mathrm{m} .7511 \mathrm{~T}>\mathrm{C}$ and in the majority of $\mathrm{m} .7511 \mathrm{~T}>\mathrm{C}$ HL patients that were reported worldwide (Table II). The AO varied from prelingual to a late postlingual usually in the third or fourth decade of life (20).

Medical records of previously reported families with the m.7511T $>\mathrm{C}$ mutation showed no other clinical features usually associated with mitochondrial diseases, i.e., muscular diseases, visual loss or neurological disorders, but few patients were diagnosed with diabetes mellitus $(17,24)$. Similarly to the French and Japanese families, some patients in our study suffered from vertigo and tinnitus (Table I). Temporal bone CT or head MRI of our patients did not reveal any significant abnormalities of the cochlear structures. At the time of writing the manuscript there was only one report on temporal bone histopathologic findings in a patient with m.7511T $>$ C mutation. In the Japanese male severe loss of spiral ganglion cells in the cochlea accompanied by severe loss of neuronal filaments in Rosenthal's canal and partial atrophy of stria vascularis in all turns of cochlea was described (22).

From the theoretical point of view, severe loss of spiral ganglion cells, as the main cause of sensorineural HL associated with the m.7511T $>\mathrm{C}$ mutation, could hinder the benefit from cochlear implantation as a sufficient number of intact ganglion cells is necessary for a CI to enable good speech discrimination (47).

$\mathrm{CI}$ is an electronic hearing prosthesis designed for individuals with profound deafness. In the newest application, electric stimulation from a CI is used in case of PD, defined as a mild to moderate low frequency SNHL sloping to a profound HL in the higher frequencies (40). In each case of cochlear implantation an individual surgical approach is required. The key issue is to avoid the inner ear trauma and consequently to prevent apoptosis (48). Therefore the correct choice of electrode and its insertion depth is essential. According to published data insertion of $16-20 \mathrm{~mm}$ is recommend (49). An individual, yet multi-featured approach is required especially in case of progressive HL, where maintaining the structure of the patient's inner ear is crucial. Typically, in case of severe HL, the preferred electrode insertion depth is $28 \mathrm{~mm}$ (47), but in case of PD the recommended insertion depth is 20-25 mm (50). Low frequencies hearing preservation after CI facilitates combining the electric and acoustic stimulation of the auditory system (40). Contrary to the theoretical assumption, results of the current study show that patient with the m.7511T $>C$ mutation and PD can have substantial benefit from CI. This findings is in line with the studies where other mtDNA (e.g., m.1555A>G) mutation were identified among successful CI users with PD $(51,52)$. Good outcomes of CI in these patients suggest that those with mutations in the mtDNA are good candidates for CI. Due to the fact that there is a huge variability in CI outcomes and many factors are thought to be involved in post-implantation performance, genetic testing would be helpful in predicting a CI benefit and facilitating the decision about treatment choices.

The main goal of this project was to search for mtDNA HL mutations, other than m.1555A $>\mathrm{G}$ and m.3243A $>\mathrm{G}$, among Polish HL patients. As there were no reports describing the prevalence of $\mathrm{m} .7511 \mathrm{~T}>\mathrm{C}$ in the Polish population so far, presence of the m.7511T $>C$ was not checked by Sanger sequencing in the analyzed patients prior to their inclusion into the NGS study, which might be considered as a limitation of this study.

In conclusion, the m.7511T $>\mathrm{C}$ mutation is an important cause of maternally inherited isolated SNHL, which may manifest from early childhood to middle age. The mutation occurs usually as homoplasmic and rarely as heteroplasmic. There is no statistically significant correlation between m.7511T $>C$ heteroplasmy level and the HL AO. The present study provides two additional HL families with the m.7511T $>$ C, which further supports the pathogenic character of the mutation and indicates how to treat such patients.

\section{Acknowledgements}

The present study was supported by National Science Centre (grant nos. 2012/05/N/NZ5/02629 and 2013/09/D/NZ5/00251).

\section{References}

1. Friedman RA, Bykhovskaya Y, Sue CM, DiMauro S, Bradley R, Fallis-Cunningham R, Paradies N, Pensak ML, Smith RJ, Groden J, et al: Maternally inherited nonsyndromic hearing loss. Am J Med Genet 84: 369-372, 1999.

2. Lightowlers RN, Taylor RW and Turnbull DM: Mutations causing mitochondrial disease: What is new and what challenges remain? Science 349: 1494-1499, 2015.

3. Van Camp G and Smith RJ: Maternally inherited hearing impairment. Clin Genet 57: 409-414, 2000.

4. Nance WE: The genetics of deafness. Ment Retard Dev Disabil Res Rev 9: 109-119, 2003. 
5. Snoeckx RL, Huygen PL, Feldmann D, Marlin S, Denoyelle F, Waligora J, Mueller-Malesinska M, Pollak A, Ploski R, Murgia A, et al: GJB2 mutations and degree of hearing loss: A multicenter study. Am J Hum Genet 77: 945-957, 2005.

6. Smith RJ, Bale JF Jr and White KR: Sensorineural hearing loss in children. Lancet 365: 879-890, 2005.

7. Hutchin TP, Thompson KR, Parker M, Newton V, BitnerGlindzicz M and Mueller RF: Prevalence of mitochondrial DNA mutations in childhood/congenital onset non-syndromal sensorineural hearing impairment. J Med Genet 38: 229-231, 2001.

8. Jacobs HT, Hutchin TP, Käppi T, Gillies G, Minkkinen K, Walker J, Thompson K, Rovio AT, Carella M, Melchionda S, et al: Mitochondrial DNA mutations in patients with postlingual, nonsyndromic hearing impairment. Eur J Hum Genet 13: 26-33, 2005 .

9. Yelverton JC, Arnos K, Xia XJ, Nance WE, Pandya A and Dodson KM: The clinical and audiologic features of hearing loss due to mitochondrial mutations. Otolaryngol Head Neck Surg 148: 1017-1022, 2013.

10. Iwanicka-Pronicka K, Pollak A, Skórka A, Lechowicz U, Korniszewski L, Westfal P, Skarżyński H and Płoski R: Audio profiles in mitochondrial deafness m.1555A $>\mathrm{G}$ and m.3243A $>\mathrm{G}$ show distinct differences. Med Sci Monit 21: 694-700, 2015.

11. Iwanicka-Pronicka K, Pollak A, Skórka A, Lechowicz U, Pajdowska M, Furmanek M, Rzeski M, Korniszewski L, Skarżyński H and Płoski R: Postlingual hearing loss as a mitochondrial 3243A $>$ G mutation phenotype. PLoS One 7: e44054, 2012.

12. Guan MX: Molecular pathogenetic mechanism of maternally inherited deafness. Ann N Y Acad Sci 1011: 259-271, 2004.

13. Kokotas H, Petersen MB and Willems PJ: Mitochondrial deafness. Clin Genet 71: 379-391, 2007.

14. Cao L, Shitara H, Horii T, Nagao Y, Imai H, Abe K, Hara T, Hayashi J and Yonekawa $\mathrm{H}$ : The mitochondrial bottleneck occurs without reduction of mtDNA content in female mouse germ cells. Nat Genet 39: 386-390, 2007

15. Taylor RW and Turnbull DM: Mitochondrial DNA mutations in human disease. Nat Rev Genet 6: 389-402, 2005.

16. Li X, Fischel-Ghodsian N, Schwartz F, Yan Q, Friedman RA and Guan MX: Biochemical characterization of the mitochondrial tRNASer (UCN) T7511C mutation associated with nonsyndromic deafness. Nucleic Acids Res 32: 867-877, 2004.

17. Ishikawa K, Tamagawa Y, Takahashi K, Kimura H, Kusakari J, Hara A and Ichimura K: Nonsyndromic hearing loss caused by a mitochondrial T7511C mutation. Laryngoscope 112: 1494-1499, 2002.

18. Chapiro E, Feldmann D, Denoyelle F, Sternberg D, Jardel C, Eliot MM, Bouccara D, Weil D, Garabédian EN, Couderc R, et al Two large French pedigrees with non syndromic sensorineural deafness and the mitochondrial DNA T7511C mutation: Evidence for a modulatory factor. Eur J Hum Genet 10: 851-856, 2002.

19. Tang X, Zheng J, Ying Z, Cai Z, Gao Y, He Z, Yu H, Yao J, Yang Y Wang H, et al: Mitochondrial tRNA (Ser(UCN)) variants in 2651 han Chinese subjects with hearing loss. Mitochondrion 23: 17-24, 2015 .

20. Yamasoba T, Tsukuda K and Suzuki M: Isolated hearing loss associated with T7511C mutation in mitochondrial DNA. Acta Otolaryngol Suppl: 13-18, 2007.

21. Chen DY, Zhu WD, Chai YC, Chen Y, Sun L, Yang T and Wu H: Mutation in PCDH15 may modify the phenotypic expression of the 7511T $>$ C mutation in MT-TS1 in a Chinese han family with maternally inherited nonsyndromic hearing loss. Int J Pediatr Otorhinolaryngol 79: 1654-1657, 2015.

22. Ishikawa K, Tamagawa Y, Takahashi K, Iino Y, Murakami Y, Kakizaki K, Kimura H, Kusakari J, Hara A and Ichimura K: Temporal bone histopathologic abnormalities associated with mitochondrial mutation T7511C. Laryngoscope 116: 1982-1986, 2006.

23. Li R, Ishikawa K, Deng JH, Heman-Ackah S, Tamagawa Y, Yang L, Bai Y, Ichimura K and Guan MX: Maternally inherited nonsyndromic hearing loss is associated with the T7511C mutation in the mitochondrial tRNASerUCN gene in a Japanese family. Biochem Biophys Res Commun 328: 32-37, 2005.

24. Sue CM, Tanji K, Hadjigeorgiou G, Andreu AL, Nishino I, Krishna S, Bruno C, Hirano M, Shanske S, Bonilla E, et al: Maternally inherited hearing loss in a large kindred with a novel T7511C mutation in the mitochondrial DNA tRNA (Ser(UCN)) gene. Neurology 52: 1905-1908, 1999.
25. Hoefsloot LH, Roux AF and Bitner-Glindzicz M; contributors to EMQN DFNB1 best practice meeting: EMQN best practice guidelines for diagnostic testing of mutations causing non-syndromic hearing impairment at the DFNB1 locus. Eur J Hum Genet 21: 1325-1329, 2013.

26. Ołdak M, Oziębło D, Pollak A, Stępniak I, Lazniewski M, Lechowicz U, Kochanek K, Furmanek M, Tacikowska G, Plewczynski D, et al: Novel neuro-audiological findings and further evidence for TWNK involvement in perrault syndrome. J Transl Med 15: 25, 2017.

27. Lorens A, Polak M, Piotrowska A and Skarzynski H: Outcomes of treatment of partial deafness with cochlear implantation: A DUET study. Laryngoscope 118: 288-294, 2008.

28. Miller SA, Dykes DD and Polesky HF: A simple salting out procedure for extracting DNA from human nucleated cells. Nucleic Acids Res 16: 1215, 1988.

29. Koressaar T and Remm M: Enhancements and modifications of primer design program primer3. Bioinforma 23: 1289-1291, 2007.

30. Untergasser A, Cutcutache I, Koressaar T, Ye J, Faircloth BC, Remm $M$ and Rozen SG: Primer3-new capabilities and interfaces. Nucleic Acids Res 40: e115, 2012.

31. Li H and Durbin R: Fast and accurate short read alignment with burrows-wheeler transform. Bioinforma 25: 1754-1760, 2009.

32. McKenna A, Hanna M, Banks E, Sivachenko A, Cibulskis K, Kernytsky A, Garimella K, Altshuler D, Gabriel S, Daly M and DePristo MA: The genome analysis toolkit: A MapReduce framework for analyzing next-generation DNA sequencing data. Genome Res 20: 1297-1303, 2010.

33. Pollak A, Lechowicz U, Kędra A, Stawiński P, Rydzanicz M, Furmanek M, Brzozowska M, Mrówka M, Skarżyński H, Skarżyński PH, et al: Novel and de novo mutations extend association of POU3F4 with distinct clinical and radiological phenotype of hearing loss. PLoS One 11: e0166618, 2016

34. Lott MT, Leipzig JN, Derbeneva O, Xie HM, Chalkia D, Sarmady M, Procaccio V and Wallace DC: mtDNA variation and analysis using mitomap and mitomaster. Curr Protoc Bioinforma 44: 1-26, 2013.

35. Robinson JT, Thorvaldsdóttir H, Winckler W, Guttman M, Lander ES, Getz G and Mesirov JP: Integrative genomics viewer. Nat Biotechnol 29: 24-26, 2011.

36. Ingman $M$ and Gyllensten U: mtDB: Human mitochondrial genome database, a resource for population genetics and medical sciences. Nucleic Acids Res 34 (Database Issue): D749-D751, 2006.

37. Weissensteiner H, Pacher D, Kloss-Brandstätter A, Forer L, Specht G, Bandelt HJ, Kronenberg F, Salas A and Schönherr S: HaploGrep 2: Mitochondrial haplogroup classification in the era of high-throughput sequencing. Nucleic Acids Res 44: W58-W63, 2016.

38. van Oven M and Kayser M: Updated comprehensive phylogenetic tree of global human mitochondrial DNA variation. Hum Mutat 30: E386-E394, 2009.

39. Free Statistics and Forecasting Software: Spearman Rank Correlation (v1.0.1). http://www.wessa.net/rwasp_spearman. wasp/. Accessed June 1, 2017.

40. Skarzyński H, Lorens A and Piotrowska A: A new method of partial deafness treatment. Med Sci Monit 9: CS20-CS24, 2003.

41. Skarzynski H, Lorens A, Piotrowska A and Skarzynski PH Hearing preservation in partial deafness treatment. Med Sci Monit 16: CR555-CR562, 2010.

42. Skarzynski H, Lorens A, Dziendziel B and Skarzynski PH: Expanding pediatric cochlear implant candidacy: A case study of electro-natural stimulation (ENS) in partial deafness treatment. Int J Pediatr Otorhinolaryngol 79: 1896-1900, 2015.

43. Skarzynski H, van de Heyning P, Agrawal S, Arauz SL, Atlas M, Baumgartner W, Caversaccio M, de Bodt M, Gavilan J, Godey B, et al: Towards a consensus on a hearing preservation classification system. Acta Otolaryngol Suppl: 3-13, 2013.

44. Skarzynski H, Matusiak M, Lorens A, Furmanek M, Pilka A and Skarzynski PH: Preservation of cochlear structures and hearing when using the nucleus slim straight (CI422) electrode in children. J Laryngol Otol 130: 332-339, 2016.

45. Rydzanicz M, Cywińska K, Wróbel M, Pollak A, Gawęcki W, Wojsyk-Banaszak I, Lechowicz U, Mueller-Malesińska M, Ołdak M, Płoski R, et al: The contribution of the mitochondrial COI/tRNA (Ser(UCN)) gene mutations to non-syndromic and aminoglycoside-induced hearing loss in polish patients. Mol Genet Metab 104: 153-159, 2011 
46. Prezant TR, Agapian JV, Bohlman MC, Bu X, Oztas S, Qiu WQ, Arnos KS, Cortopassi GA, Jaber L, Rotter JI, et al: Mitochondrial ribosomal RNA mutation associated with both antibiotic-induced and non-syndromic deafness. Nat Genet 4: 289-294, 1993

47. Skarzynski H, Lorens A, Zgoda M, Piotrowska A, Skarzynski PH and Szkielkowska A: Atraumatic round window deep insertion of cochlear electrodes. Acta Otolaryngol 131: 740-749, 2011.

48. De Seta D, Torres R, Russo FY, Ferrary E, Kazmitcheff G, Heymann D, Amiaud J, Sterkers O, Bernardeschi D and Nguyen Y: Damage to inner ear structure during cochlear implantation: Correlation between insertion force and radio-histological findings in temporal bone specimens. Hear Res 344: 90-97, 2017.

49. Gantz BJ, Dunn C, Walker E, Van Voorst T, Gogel S and Hansen M: Outcomes of adolescents with a short electrode cochlear implant with preserved residual hearing. Otol Neurotol 37: e118-e125, 2016.
50. Nordfalk KF, Rasmussen K, Bunne $\mathrm{M}$ and Jablonski GE: Deep round window insertion versus standard approach in cochlear implant surgery. Eur Arch Otorhinolaryngol 273: 43-50, 2016.

51. Usami S, Miyagawa M, Nishio SY, Moteki H, Takumi Y, Suzuki M, Kitano Y and Iwasaki S: Patients with CDH23 mutations and the $1555 \mathrm{~A}>\mathrm{G}$ mitochondrial mutation are good candidates for electric acoustic stimulation (EAS). Acta Otolaryngol 132: 377-384, 2012.

52. Miyagawa M, Nishio SY and Usami S: A comprehensive study on the etiology of patients receiving cochlear implantation with special emphasis on genetic epidemiology. Otol Neurotol 37: e126-e134, 2016

(c) (1) This work is licensed under a Creative Commons cc) Attribution-NonCommercial-NoDerivatives 4.0 International (CC BY-NC-ND 4.0) License. 